\title{
Comparative Renal Effects of Dipeptidyl Peptidase-4 Inhibitors and Sodium-Glucose Cotransporter 2 Inhibitors on Individual Outcomes in Patients with Type 2 Diabetes: A Systematic Review and Network Meta-Analysis
}

\author{
Jae Hyun Bae ${ }^{1, *}$, Eun-Gee Park ${ }^{2, *}$, Sunhee Kim², Sin Gon Kim ${ }^{1}$, Seokyung Hahn" ${ }^{3,4,5}$, Nam Hoon Kim ${ }^{1}$ \\ ${ }^{1}$ Department of Internal Medicine, Korea University Anam Hospital, Korea University College of Medicine; ${ }^{2}$ Interdisciplinary \\ Program in Medical Informatics, Seoul National University College of Medicine; ${ }^{3}$ Division of Medical Statistics, Medical \\ Research Collaborating Center, Seoul National University Hospital; ${ }^{4}$ Department of Human Systems Medicine, Seoul National \\ University College of Medicine; ${ }^{5}$ Institute of Health Policy and Management, Medical Research Center, Seoul National \\ University, Seoul, Korea
}

Background: To compare the renal effects of dipeptidyl peptidase-4 (DPP-4) inhibitors and sodium-glucose cotransporter 2 (SGLT2) inhibitors on individual outcomes in patients with type 2 diabetes.

Methods: We searched electronic databases (MEDLINE, Embase, and the Cochrane Central Register of Controlled Trials) from inception to June 2019 to identity eligible randomized controlled trials of DPP-4 inhibitors or SGLT2 inhibitors that reported at least one kidney outcome in patients with type 2 diabetes. Outcomes of interest were microalbuminuria, macroalbuminuria, worsening nephropathy, and end-stage kidney disease (ESKD). We performed an arm-based network meta-analysis using Bayesian methods and calculated absolute risks and rank probabilities of each treatment for the outcomes.

Results: Seventeen studies with 87,263 patients were included. SGLT2 inhibitors significantly lowered the risks of individual kidney outcomes, including microalbuminuria (odds ratio [OR], 0.64; 95\% credible interval [CrI], 0.41 to 0.93 ), macroalbuminuria (OR, 0.48 ; $95 \% \mathrm{CrI}, 0.24$ to 0.72 ), worsening nephropathy (OR, $0.65 ; 95 \%$ CrI, 0.44 to 0.91 ), and ESKD (OR, $0.65 ; 95 \%$ CrI, 0.46 to 0.98 ) as compared with placebo. However, DPP-4 inhibitors did not lower the risks. SGLT2 inhibitors were considerably associated with higher absolute risk reductions in all kidney outcomes than DPP-4 inhibitors, although the benefits were statistically insignificant. The rank probabilities showed that SGLT2 inhibitors were better treatments for lowering the risk of albuminuria and ESKD than placebo or DPP-4 inhibitors.

Conclusion: SGLT2 inhibitors were superior to DPP-4 inhibitors in reducing the risk of albuminuria and ESKD in patients with type 2 diabetes.

Received: 12 November 2020, Revised: 14 January 2021,

Accepted: 15 February 2021

Corresponding authors: Nam Hoon Kim

Department of Internal Medicine, Korea University College of Medicine, 73 Goryeodae-ro, Seongbuk-gu, Seoul 02841, Korea

Tel: +82-2-920-6268, Fax: +82-2-953-9355, E-mail: pourlife@korea.ac.kr

Seokyung Hahn

Department of Human Systems Medicine, Seoul National University College of

Medicine, 103 Daehak-ro, Jongno-gu, Seoul 03080, Korea

Tel: +82-2-740-8911, Fax: +82-2-743-8361, E-mail: hahns@snu.ac.kr

\section{Copyright $(92021$ Korean Endocrine Society}

This is an Open Access article distributed under the terms of the Creative Commons Attribution Non-Commercial License (https://creativecommons.org/ licenses/by-nc/4.0/) which permits unrestricted non-commercial use, distribution, and reproduction in any medium, provided the original work is properly cited.

*These authors contributed equally to this work. 
Keywords: Albuminuria; Diabetes mellitus, type 2; Diabetic nephropathies; Dipeptidyl-peptidase IV inhibitors; Kidney failure, chronic; Network meta-analysis; Sodium-glucose transporter 2 inhibitors; Systematic review

\section{INTRODUCTION}

Type 2 diabetes is a main cause of chronic kidney disease (CKD) worldwide [1]. The prevalence of diabetic kidney disease has been reported to be $38 \%$ to $68 \%$ and is gradually increasing with the global diabetes epidemic [2,3]. Since CKD is closely related to a high risk of morbidity and mortality in type 2 diabetes [4-6], appropriate interventions should be integrated into clinical practice for preventing its development and progression [7].

On the basis of the results from cardiovascular outcome trials, current guidelines for type 2 diabetes prioritize the use of sodium-glucose cotransporter 2 (SGLT2) inhibitors in patients with CKD [8-10]. Four large randomized controlled trials (RCTs) have proven that canagliflozin, dapagliflozin, and empagliflozin are beneficial for lowering the risk of hard kidney outcomes compared with placebo [11-14], although three of them [11-13] assessed these outcomes as secondary endpoints. On the other hand, incretin-based drugs have also been reported to be potentially renoprotective in many, but not all, clinical trials for type 2 diabetes [15-18]. Saxagliptin decreased urine albumin-to-creatinine ratio (UACR) regardless of glycemic control in patients with established cardiovascular disease or multiple cardiovascular risk factors [17]. Linagliptin also reduced albuminuria progression in patients with CKD [18]. To date, dipeptidyl peptidase-4 (DPP-4) inhibitors seem to have beneficial renal effects on surrogate markers rather than hard outcomes, especially in the early stages of CKD.

Hence we had a question about whether SGLT2 inhibitors had a comparative advantage over DPP-4 inhibitors for kidney outcomes, especially in the early stages of CKD. Given that there are only a few head-to-head trials of those two classes of drugs, combining evidence from direct and indirect comparisons can provide convincing results. In this regard, we performed a systematic review and network meta-analysis of RCTs to compare the renal effects of DPP-4 inhibitors and SGLT2 inhibitors on individual outcomes in patients with type 2 diabetes.

\section{METHODS}

This study was performed according to a prespecified protocol
(Appendix S1). The results were reported by the Preferred Reporting Items for Systematic Review and Meta-analyses (PRISMA) extension statement for reporting of systematic reviews incorporating network meta-analyses of healthcare interventions (Supplemental Table S1) [19].

\section{Data sources and search strategy}

The search strategy for the present study has been reported previously [20,21]. Briefly, MEDLINE, Embase, and the Cochrane Central Register of Controlled Trials were systematically searched for RCTs of DPP-4 inhibitors or SGLT2 inhibitors (Appendix S1). We retrieved full-text articles without restrictions of language and publication status. Initially, the search period was set from inception to September 2017 but later extended until June 2019 to include more relevant data. We also searched for presentations from scientific conferences to obtain information not described in published articles.

\section{Study selection}

We identified eligible trials that compared DPP-4 inhibitors or SGLT2 inhibitors with placebo and/or other antidiabetic drugs in patients with type 2 diabetes. The inclusion criteria were studies that (1) were performed for $\geq 12$ weeks; (2) reported at least one kidney outcome, including microalbuminuria, macroalbuminuria, end-stage kidney disease (ESKD), dialysis, or kidney transplantation. We removed duplicated records and screened titles and abstracts. The details are described in Appendix S1.

\section{Data extraction}

The procedure of data extraction has been detailed elsewhere $[20,21]$. Two authors (J.H.B. and E.G.P.) conducted a standardized data extraction independently (Appendix S1). Outcomes of interest were microalbuminuria (UACR $>30 \mathrm{mg} / \mathrm{g}$ ), macroalbuminuria (UACR $>300 \mathrm{mg} / \mathrm{g}$ ), worsening nephropathy (developing microalbuminuria or macroalbuminuria from normoalbuminuria, or progression from microalbuminuria to macroalbuminuria), and ESKD. The number of study participants reporting kidney outcomes was extracted along with study duration, intervention, comparator, and background antidiabetic drugs. 


\section{Quality assessment}

Study quality and risk of bias were assessed using the Cochrane Risk of Bias Tool [22]. Four authors (J.H.B., E.G.P., S.K., and N.H.K.) reviewed the studies and evaluated the risk of bias as adequate (low), unclear, or inadequate (high) according to the following domains: random sequence generation, allocation concealment, blinding of participants and personnel, blinding of outcome assessment, incomplete outcome data, selective reporting, and other sources of bias.

\section{Data synthesis and statistical analysis}

Pairwise meta-analyses were conducted using a fixed effect model for direct comparisons of intervention and comparator (DPP-4 inhibitor vs. placebo, SGLT2 inhibitor vs. placebo, and SGLT2 inhibitor vs. DPP-4 inhibitor, respectively). We evaluated statistical heterogeneity using the $I^{2}$ statistic, $\tau^{2}$ statistic, and Cochran's $Q$ test [23]. To combine the direct and indirect estimates of binary outcomes, we performed an arm-based network meta-analysis using Bayesian methods [24]. Before the network meta-analysis, we used a back-calculation method with a fixed effect model to check inconsistency between direct and indirect estimates [25]. We also checked homogeneity in the results of the placebo groups for each outcome. A subgroup analysis was conducted to estimate the risk of ESKD in studies with a duration of $\geq 52$ weeks. The result of pairwise and network metaanalyses was reported as median odds ratio (OR) and its 95\% credible interval (CrI). Additionally, the posterior densities of absolute risks and rank probabilities were calculated to confirm the best treatments for each kidney outcome. Statistical analyses were performed using $\mathrm{R}$ version 3.1.0 (R Foundation for Statistical Computing, Vienna, Austria). $P$ values $<0.05$ and $<0.10$ were considered as statistically significant for the outcomes and test for heterogeneity, respectively.

\section{Ethical statement}

Ethical approval is not required because this study extracted and synthesized data from previously published articles.

\section{RESULTS}

\section{Study characteristics and network geometry}

A flow diagram of the study screening and selection is depicted in Supplemental Fig. S1. Of 7,979 initially identified and six additional records, 17 RCTs with 18 publications involving 87,263 patients were finally included: 22,074, 24,262, and 40,943 were the DPP-4 inhibitor, SGLT2 inhibitor, and placebo groups, re- spectively. Two publications [26,27] reported different kidney outcomes from the same trial. One publication [28] was a pooled analysis of five RCTs [29-33]. The baseline characteristics of the studies are summarized in Table $1[11,13,14,17,18$, 26-28,30-39]. The number of study participants ranged from 145 to 17,160 . The study duration was varied from 52 weeks to a median of 4.2 years. The mean estimated glomerular filtration rate (eGFR) ranged from 43 to $92 \mathrm{~mL} / \mathrm{min} / 1.73 \mathrm{~m}^{2}$ across the studies. Fig. 1 shows the network of treatment comparisons for each kidney outcome.

\section{Study quality and risk of bias}

Supplemental Fig. S2 shows the risk of bias of the studies. Random sequence generation and allocation concealment were not reported in two studies $[28,34]$. All studies presented adequate blinding of participants, personnel, and outcome assessment. Four studies [17,28,30,32] had incomplete outcome data owing to losses to follow-up. The possibility of selective reporting was found in one study [28] because of a pooled analysis.

\section{Development and progression of albuminuria}

A total of nine RCTs evaluated the effects of the drugs on albuminuria (Supplemental Fig. S3A-C). As compared with placebo, SGLT2 inhibitors significantly lowered the risks of microalbuminuria (OR, $0.64 ; 95 \% \mathrm{CrI}, 0.41$ to 0.93 ), macroalbuminuria (OR, $0.48 ; 95 \% \mathrm{CrI}, 0.24$ to 0.72 ), and worsening nephropathy (OR, 0.65; 95\% CrI, 0.44 to 0.91), whereas DPP-4 inhibitors did not lower the risks. SGLT2 inhibitors did not lower the risks of microalbuminuria (OR, $0.80 ; 95 \% \mathrm{CrI}, 0.48$ to 1.37 ), macroalbuminuria (OR, $0.59 ; 95 \% \mathrm{CrI}, 0.27$ to 1.07 ), and worsening nephropathy (OR, $0.79 ; 95 \% \mathrm{CrI}, 0.50$ to 1.36 ) compared with DPP-4 inhibitors (Table 2, Supplemental Fig. S3A-C). However, SGLT2 inhibitors showed a higher absolute risk reduction in albuminuria and ranked as the best treatment (Fig. 2A-C). These results were more pronounced for macroalbuminuria than for microalbuminuria. Heterogeneity was regarded as considerable only across the studies comparing SGLT2 inhibitors and placebo (Supplemental Fig. S3A-C).

\section{Development of ESKD}

Thirteen RCTs assessed ESKD events as an outcome (Supplemental Fig. S3D). SGLT2 inhibitors significantly lowered the risk of ESKD compared with placebo (OR, 0.65; 95\% CrI, 0.46 to 0.98 ). By contrast, DPP-4 inhibitors did not affect the risk of ESKD (OR, 0.97; 95\% CrI, 0.71 to 1.40 ). In indirect comparisons, SGLT2 inhibitors insignificantly lowered the risk of 







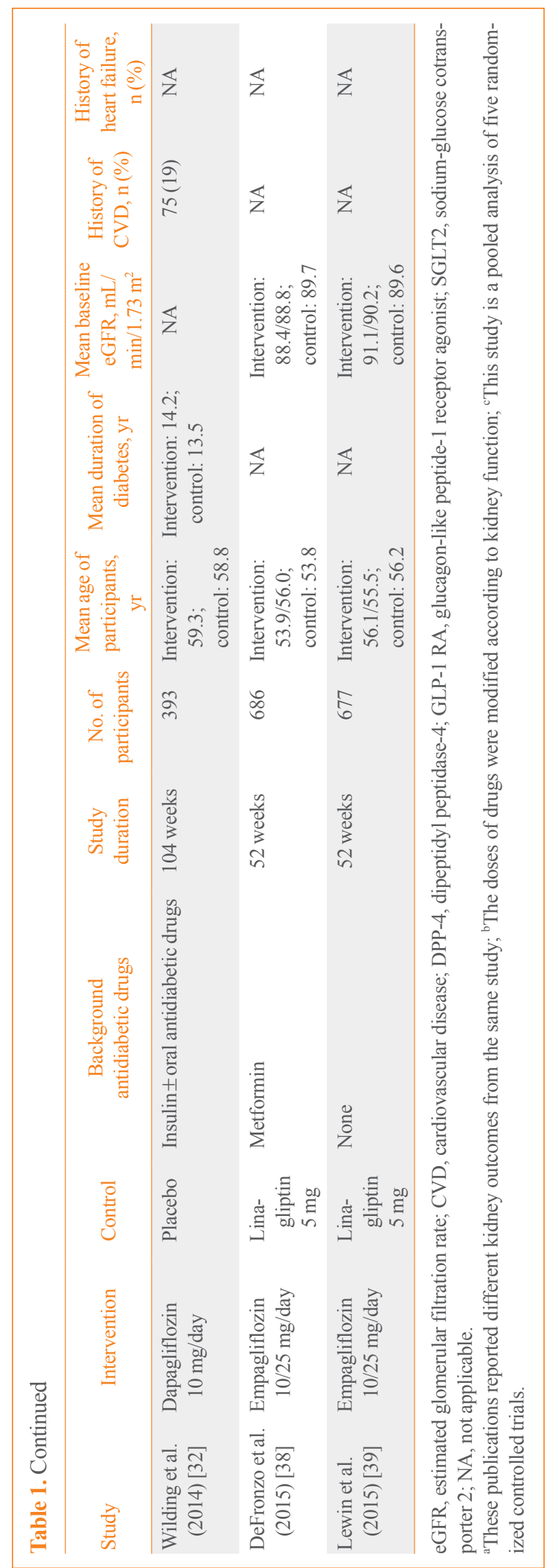

Table 2. Results of the Network Meta-Analysis for Individual Kidney Outcomes

\begin{tabular}{|c|c|c|c|}
\hline Variable & Placebo & $\begin{array}{c}\text { DPP-4 } \\
\text { inhibitors }\end{array}$ & $\begin{array}{c}\text { SGLT2 } \\
\text { inhibitors }\end{array}$ \\
\hline \multicolumn{4}{|l|}{ Microalbuminuria } \\
\hline Placebo & 1 & & \\
\hline DPP-4 inhibitors & $0.80(0.46-1.24)$ & 1 & \\
\hline SGLT2 inhibitors & $0.64(0.41-0.93)$ & $0.80(0.48-1.37)$ & 1 \\
\hline \multicolumn{4}{|l|}{ Macroalbuminuria } \\
\hline Placebo & 1 & & \\
\hline DPP-4 inhibitors & $0.82(0.44-1.33)$ & 1 & \\
\hline SGLT2 inhibitors & $0.48(0.24-0.72)$ & $0.59(0.27-1.07)$ & 1 \\
\hline \multicolumn{4}{|c|}{ Worsening nephropathy } \\
\hline Placebo & 1 & & \\
\hline DPP-4 inhibitors & $0.82(0.47-1.23)$ & 1 & \\
\hline SGLT2 inhibitors & $0.65(0.44-0.91)$ & $0.79(0.50-1.36)$ & 1 \\
\hline \multicolumn{4}{|c|}{ End-stage kidney disease } \\
\hline Placebo & 1 & & \\
\hline DPP-4 inhibitors & $0.97(0.71-1.40)$ & 1 & \\
\hline SGLT2 inhibitors & $0.65(0.46-0.98)$ & $0.67(0.42-1.11)$ & 1 \\
\hline
\end{tabular}

Values are expressed as median odds ratios ( $95 \%$ credible intervals). DPP-4, dipeptidyl peptidase-4; SGLT2, sodium-glucose cotransporter 2.

ESKD (OR, 0.67; 95\% CrI, 0.42 to 1.11 ) compared with DPP-4 inhibitors (Table 2). However, the subgroup analysis showed that SGLT2 inhibitors were associated with a significantly lower risk of ESKD than DPP-4 inhibitors (OR, 0.60; 95\% CrI, 0.34 to 0.91 ) in the studies longer than 52 weeks (Supplemental Table S2). No studies which directly compared the risk of ESKD between the two drugs were found.

SGLT2 inhibitors showed a higher absolute risk reduction in ESKD than placebo and DPP-4 inhibitors, and thus ranked as the best treatment (Fig. 2D). Heterogeneity was not significant across the studies included in this analysis (Supplemental Fig. S3D). The subgroup analysis revealed similar results for the studies longer than 52 weeks (Supplemental Fig. S4).

\section{Checking consistency in network meta-analysis}

The results from checking inconsistency for the network metaanalysis are presented in Supplemental Table S3. There was no substantial inconsistency between the direct and indirect estimates in the network meta-analysis for microalbuminuria ( $P=0.83)$, macroalbuminuria $(P=0.99)$, and worsening nephropathy $(P=0.69)$. For ESKD, a statistical inconsistency check was not possible because there was no study of a direct 


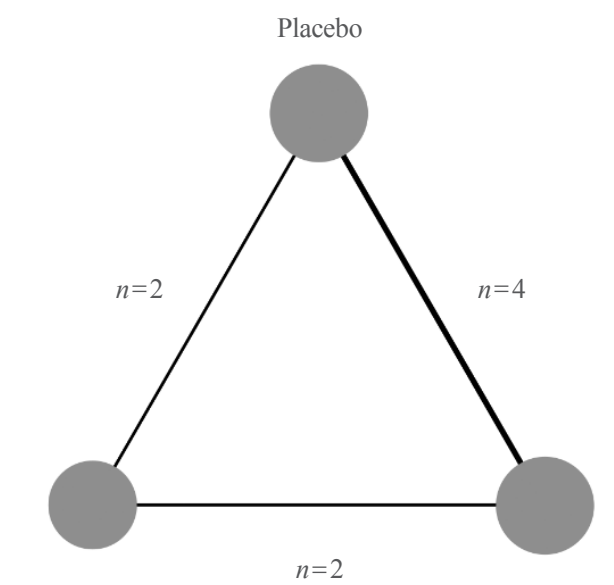

DPP-4 inhibitors

SGLT2 inhibitors

(A)



DPP-4 inhibitors

SGLT2 inhibitors

B

Fig. 1. Network of the treatment comparisons for (A) microalbuminuria, macroalbuminuria, worsening nephropathy, and (B) end-stage kidney disease. Node size is proportional to the number of studies. Lines indicate direct comparisons between the treatments, and their thickness corresponds to the number of studies in each comparison. DPP-4, dipeptidyl peptidase-4; SGLT2, sodium-glucose cotransporter 2.
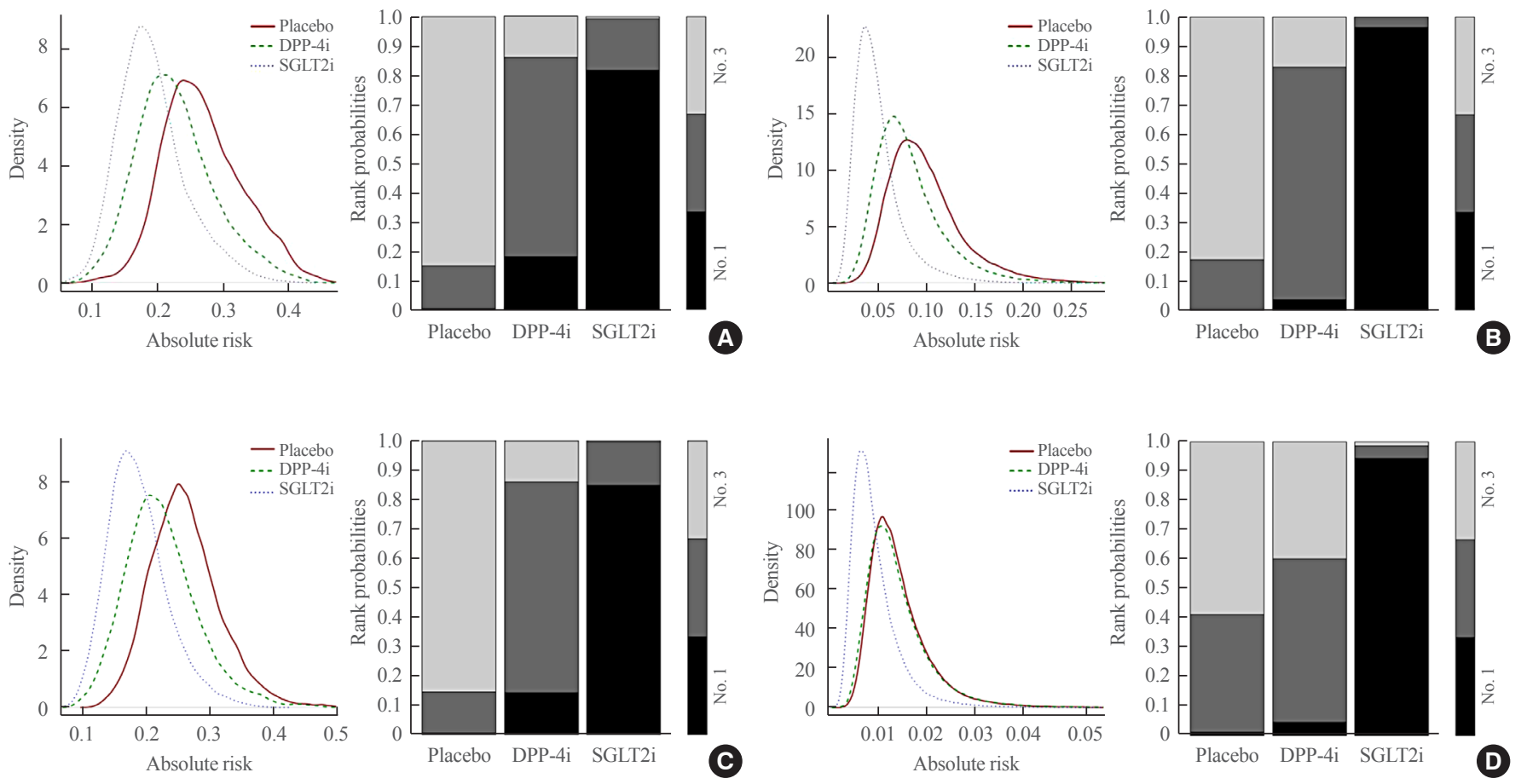

Fig. 2. Absolute risks and rank probabilities of the treatments for (A) microalbuminuria, (B) macroalbuminuria, (C) worsening nephropathy, and (D) end-stage kidney disease. Ranking (no. 1 to no. 3) represents the best, second best, and third best treatment for reducing the risk of each outcome, respectively. DPP-4i, dipeptidyl peptidase-4 inhibitor; SGLT2i, sodium-glucose cotransporter 2 inhibitor.

comparison between DPP-4 inhibitor and SGLT2 inhibitor for the outcome in this network. On the other hand, we noted an outlying result from one study of SGLT2 inhibitor (Canagliflozin and Renal Events in Diabetes with Established Nephropathy Clinical Evaluation [CREDENCE] trial) [14], with a relatively larger proportion of ESKD events in the placebo group (Supplemental Fig. S5), probably owing to the characteristics of participants who were at high risk of ESKD. However, since a high event rate was also observed in the SGLT2 inhibitor group, this study did not alter direction or heterogeneity in 
the treatment effect measure (Supplemental Fig. S3D). Sensitivity analysis with exclusion of the study also demonstrated similar results in absolute risks and rank probabilities (Supplemental Table S4, Supplemental Fig. S6).

\section{DISCUSSION}

This network meta-analysis demonstrated that SGLT2 inhibitors were more beneficial for reducing the risk of albuminuria and ESKD than DPP-4 inhibitors in patients with type 2 diabetes. Although the benefits were statistically insignificant, SGLT2 inhibitors had higher absolute risk reductions and ranked as better treatments for all of the individual outcomes than DPP-4 inhibitors.

We found that SGLT2 inhibitors were the most effective treatment for lowering the risk of albuminuria, especially macroalbuminuria, followed by DPP-4 inhibitors and placebo. Given that glucose-lowering efficacy is generally similar between SGLT2 inhibitors and DPP-4 inhibitors [40], the benefits might be owing to the effects beyond glycemic control. As reviewed elsewhere [41], SGLT2 inhibitor could reduce albuminuria by hemodynamic and nonhemodynamic mechanisms. It has been suggested that restoration of tubuloglomerular feedback, which leads to a reduction in intraglomerular pressure, is mainly responsible for the favorable renal effects [42]. In addition, body weight reduction, decrease in systemic blood pressure and vascular stiffness, amelioration of inflammation, fibrosis, and oxidative stress, and reduction in renal workload could improve albuminuria [41]. Several studies reported that DPP-4 inhibitors exerted anti-inflammatory [43], antifibrotic [44], and anti-atherosclerotic [45] properties as well as improved the endothelial function via glucagon-like peptide-1 (GLP-1)-related pathways $[46,47]$. However, unlike SGLT2 inhibitors, natriuresis and diuresis induced by DPP-4 inhibitors were reduced in type 2 diabetes [48] and caused no significant hemodynamic changes [49]. Moreover, a decrease in the degradation of neuropeptide $Y$ by DPP-4 inhibition promoted sympathetic activation and vasoconstriction via the $\mathrm{Y} 1$ receptor in patients using angiotensinconverting enzyme inhibitor (ACEI) or angiotensin receptor blocker (ARB) [50]. In the present study, approximately $80 \%$ of patients in the DPP-4 inhibitor group were receiving ACEIs/ ARBs, and the interaction between these medications could negate the beneficial renal effects. Meanwhile, SGLT2 inhibitor prevented the progression of CKD irrespective of antihypertensive therapy, including ACEIs/ARBs [51]. Therefore, SGLT2 inhibitors could have greater albuminuria-lowering effects than
DPP-4 inhibitors by altering renal and systemic hemodynamics.

SGLT2 inhibitors reduced the risk of ESKD compared with DPP-4 inhibitors or placebo, whereas DPP-4 inhibitors did not lower the risk. Cardiovascular outcome trials have shown that SGLT2 inhibitors consistently reduce the composite of doubling of serum creatinine, ESKD, and death from kidney disease by $34 \%$ to $47 \%[11,12,14,52]$ in both primary and secondary prevention populations [53]. The direct renal hemodynamic effects of these drugs play an important role in slowing the progression of CKD [41]. In our study, the reduction in the risk of ESKD was more pronounced with a longer duration of SGLT2 inhibitor treatment. SGLT2 inhibitors preserved the renoprotective effect even in patients with a low eGFR irrespective of their metabolic effects [42]. A recent meta-analysis suggested that SGLT2 inhibitors produced a greater improvement in kidney outcomes in patients with a higher baseline eGFR [53]. These findings indicate that long-term use of SGLT2 inhibitors from the early stage of CKD is important to delay its progression. On the other hand, DPP-4 inhibitors have favorable effects on risk factors for ESKD, including hyperglycemia [54] and albuminuria [17,18]. Nevertheless, DPP-4 inhibitors have not shown any benefits in ESKD [20]. In the Cardiovascular and Renal Microvascular Outcome Study with Linagliptin (CARMELINA) trial, linagliptin decreased the risk of albuminuria progression without affecting a decline in eGFR, ESKD, or death from kidney disease [18]. The beneficial effects of incretin-based drugs on kidney outcomes have been reported in patients with GLP-1 receptor agonists but not in those with DPP-4 inhibitors [55]. The benefits were mainly attributed to a reduction in the progression of albuminuria in patients with relatively normal kidney function [55]. Aside from GLP-1, the complexity of DPP-4 actions on its substrate, such as stromal cell-derived factor-1 $\alpha$ [56-58], might influence the effects of DPP-4 inhibitors on the kidney $[59,60]$. Consequently, SGLT2 inhibitors lowered the risk of ESKD in a broad range of patients with type 2 diabetes as compared with DPP-4 inhibitors.

The present study has several limitations. First, most of the DPP-4 inhibitor studies did not evaluate kidney outcomes as prespecified endpoints. Second, RCTs that directly compared the effects of DPP-4 inhibitors and SGLT2 inhibitors on ESKD were not identified. Therefore, the results for ESKD were from indirect comparisons, which requires careful interpretation. Finally, more detailed analyses according to the baseline risk of CKD could not be conducted owing to the small number of studies and insufficient information.

In conclusion, our network meta-analysis showed that SGLT2 
inhibitors were superior to DPP-4 inhibitors in reducing the risk of albuminuria and ESKD in patients with type 2 diabetes. This study warrants further investigation to directly compare the effects of the two classes of drugs on kidney outcomes.

\section{CONFLICTS OF INTEREST}

No potential conflict of interest relevant to this article was reported.

\section{AUTHOR CONTRIBUTIONS}

Conception or design: J.H.B., E.G.P., S.H., N.H.K. Acquisition, analysis, or interpretation of data: J.H.B., E.G.P., S.K., S.G.K., S.H., N.H.K. Drafting the work or revising: J.H.B., E.G.P., S.H., N.H.K. Final approval of the manuscript: J.H.B., E.G.P., S.K., S.G.K., S.H., N.H.K.

\section{ORCID}

Jae Hyun Bae https://orcid.org/0000-0002-1384-6123

Eun-Gee Park https://orcid.org/0000-0001-9408-9584

Seokyung Hahn https://orcid.org/0000-0002-4684-4917

Nam Hoon Kim https://orcid.org/0000-0002-9926-1344

\section{REFERENCES}

1. Webster AC, Nagler EV, Morton RL, Masson P. Chronic kidney disease. Lancet 2017;389:1238-52.

2. Thomas MC, Brownlee M, Susztak K, Sharma K, JandeleitDahm KA, Zoungas S, et al. Diabetic kidney disease. Nat Rev Dis Primers 2015;1:15018.

3. International Diabetes Federation. IDF diabetes atlas. 9th ed. Brussels: International Diabetes Federation; 2019.

4. Tonelli M, Wiebe N, Culleton B, House A, Rabbat C, Fok M, et al. Chronic kidney disease and mortality risk: a systematic review. J Am Soc Nephrol 2006;17:2034-47.

5. Afkarian M, Sachs MC, Kestenbaum B, Hirsch IB, Tuttle $\mathrm{KR}$, Himmelfarb J, et al. Kidney disease and increased mortality risk in type 2 diabetes. J Am Soc Nephrol 2013;24: 302-8.

6. Toyama T, Furuichi K, Ninomiya T, Shimizu M, Hara A, Iwata $\mathrm{Y}$, et al. The impacts of albuminuria and low eGFR on the risk of cardiovascular death, all-cause mortality, and renal events in diabetic patients: meta-analysis. PLoS One 2013;8:e71810.
7. Kidney Disease: Improving Global Outcomes (KDIGO) CKD Work Group. Summary of recommendation statements. Kidney Int Suppl (2011) 2013;3:5-14.

8. Davies MJ, D’Alessio DA, Fradkin J, Kernan WN, Mathieu C, Mingrone G, et al. Management of hyperglycemia in type 2 diabetes, 2018. A consensus report by the American Diabetes Association (ADA) and the European Association for the Study of Diabetes (EASD). Diabetes Care 2018;41:2669-701.

9. Garber AJ, Abrahamson MJ, Barzilay JI, Blonde L, Bloomgarden ZT, Bush MA, et al. Consensus statement by the American Association of Clinical Endocrinologists and American College of Endocrinology on the comprehensive type 2 diabetes management algorithm: 2019 executive summary. Endocr Pract 2019;25:69-100.

10. Kim MK, Ko SH, Kim BY, Kang ES, Noh J, Kim SK, et al. 2019 Clinical practice guidelines for type 2 diabetes mellitus in Korea. Diabetes Metab J 2019;43:398-406.

11. Wanner C, Inzucchi SE, Lachin JM, Fitchett D, von Eynatten M, Mattheus M, et al. Empagliflozin and progression of kidney disease in type 2 diabetes. N Engl J Med 2016;375: 323-34.

12. Neal B, Perkovic V, Mahaffey KW, de Zeeuw D, Fulcher G, Erondu N, et al. Canagliflozin and cardiovascular and renal events in type 2 diabetes. N Engl J Med 2017;377:644-57.

13. Mosenzon O, Wiviott SD, Cahn A, Rozenberg A, Yanuv I, Goodrich EL, et al. Effects of dapagliflozin on development and progression of kidney disease in patients with type 2 diabetes: an analysis from the DECLARE-TIMI 58 randomised trial. Lancet Diabetes Endocrinol 2019;7:606-17.

14. Perkovic V, Jardine MJ, Neal B, Bompoint S, Heerspink HJL, Charytan DM, et al. Canagliflozin and renal outcomes in type 2 diabetes and nephropathy. N Engl J Med 2019;380: 2295-306.

15. Mann JFE, Orsted DD, Brown-Frandsen K, Marso SP, Poulter NR, Rasmussen S, et al. Liraglutide and renal outcomes in type 2 diabetes. N Engl J Med 2017;377:839-48.

16. Gerstein HC, Colhoun HM, Dagenais GR, Diaz R, Lakshmanan M, Pais P, et al. Dulaglutide and renal outcomes in type 2 diabetes: an exploratory analysis of the REWIND randomised, placebo-controlled trial. Lancet 2019;394:131-8.

17. Mosenzon O, Leibowitz G, Bhatt DL, Cahn A, Hirshberg B, Wei C, et al. Effect of saxagliptin on renal outcomes in the SAVOR-TIMI 53 trial. Diabetes Care 2017;40:69-76.

18. Rosenstock J, Perkovic V, Johansen OE, Cooper ME, Kahn SE, Marx N, et al. Effect of linagliptin vs placebo on major cardiovascular events in adults with type 2 diabetes and high 
cardiovascular and renal risk: the CARMELINA randomized clinical trial. JAMA 2019;321:69-79.

19. Hutton B, Salanti G, Caldwell DM, Chaimani A, Schmid $\mathrm{CH}$, Cameron $\mathrm{C}$, et al. The PRISMA extension statement for reporting of systematic reviews incorporating network metaanalyses of health care interventions: checklist and explanations. Ann Intern Med 2015;162:777-84.

20. Bae JH, Kim S, Park EG, Kim SG, Hahn S, Kim NH. Effects of dipeptidyl peptidase- 4 inhibitors on renal outcomes in patients with type 2 diabetes: a systematic review and meta-analysis. Endocrinol Metab (Seoul) 2019;34:80-92.

21. Bae JH, Park EG, Kim S, Kim SG, Hahn S, Kim NH. Effects of sodium-glucose cotransporter 2 inhibitors on renal outcomes in patients with type 2 diabetes: a systematic review and meta-analysis of randomized controlled trials. Sci Rep 2019;9:13009.

22. Higgins JP, Altman DG. Cochrane handbook for systematic reviews of interventions. Chichester: John Wiley \& Sons Ltd; 2008. Chapter 8, Assessing risk of bias in included studies; p. 187-242.

23. Deeks JJ, Higgins JP, Altman DG. Cochrane handbook for systematic reviews of interventions. Chichester, UK: John Wiley \& Sons Ltd; 2008. Chapter 9, Analysing data and undertaking meta-analyses; p. 243-96.

24. Zhang J, Carlin BP, Neaton JD, Soon GG, Nie L, Kane R, et al. Network meta-analysis of randomized clinical trials: reporting the proper summaries. Clin Trials 2014;11:246-62.

25. Dias S, Welton NJ, Caldwell DM, Ades AE. Checking consistency in mixed treatment comparison meta-analysis. Stat Med 2010;29:932-44.

26. Green JB, Bethel MA, Armstrong PW, Buse JB, Engel SS, Garg J, et al. Effect of sitagliptin on cardiovascular outcomes in type 2 diabetes. N Engl J Med 2015;373:232-42.

27. Cornel JH, Bakris GL, Stevens SR, Alvarsson M, Bax WA, Chuang LM, et al. Effect of sitagliptin on kidney function and respective cardiovascular outcomes in type 2 diabetes: outcomes from TECOS. Diabetes Care 2016;39:2304-10.

28. Kosiborod M, Gause-Nilsson I, Xu J, Sonesson C, Johnsson E. Efficacy and safety of dapagliflozin in patients with type 2 diabetes and concomitant heart failure. J Diabetes Complications 2017;31:1215-21.

29. Strojek K, Yoon KH, Hruba V, Elze M, Langkilde AM, Parikh S. Effect of dapagliflozin in patients with type 2 diabetes who have inadequate glycaemic control with glimepiride: a randomized, 24-week, double-blind, placebo-controlled trial. Diabetes Obes Metab 2011;13:928-38.
30. Kohan DE, Fioretto P, Tang W, List JF. Long-term study of patients with type 2 diabetes and moderate renal impairment shows that dapagliflozin reduces weight and blood pressure but does not improve glycemic control. Kidney Int 2014;85: 962-71.

31. Leiter LA, Cefalu WT, de Bruin TW, Gause-Nilsson I, Sugg J, Parikh SJ. Dapagliflozin added to usual care in individuals with type 2 diabetes mellitus with preexisting cardiovascular disease: a 24-week, multicenter, randomized, doubleblind, placebo-controlled study with a 28 -week extension. J Am Geriatr Soc 2014;62:1252-62.

32. Wilding JP, Woo V, Rohwedder K, Sugg J, Parikh S; Dapagliflozin 006 Study Group. Dapagliflozin in patients with type 2 diabetes receiving high doses of insulin: efficacy and safety over 2 years. Diabetes Obes Metab 2014;16:124-36.

33. Cefalu WT, Leiter LA, de Bruin TW, Gause-Nilsson I, Sugg J, Parikh SJ. Dapagliflozin's effects on glycemia and cardiovascular risk factors in high-risk patients with type 2 diabetes: a 24-week, multicenter, randomized, double-blind, placebo-controlled study with a 28 -week extension. Diabetes Care 2015;38:1218-27.

34. White WB, Cannon CP, Heller SR, Nissen SE, Bergenstal RM, Bakris GL, et al. Alogliptin after acute coronary syndrome in patients with type 2 diabetes. N Engl J Med 2013; 369:1327-35.

35. Bailey CJ, Morales Villegas EC, Woo V, Tang W, Ptaszynska A, List JF. Efficacy and safety of dapagliflozin monotherapy in people with type 2 diabetes: a randomized doubleblind placebo-controlled 102-week trial. Diabet Med 2015; 32:531-41.

36. Barnett AH, Mithal A, Manassie J, Jones R, Rattunde H, Woerle HJ, et al. Efficacy and safety of empagliflozin added to existing antidiabetes treatment in patients with type 2 diabetes and chronic kidney disease: a randomised, doubleblind, placebo-controlled trial. Lancet Diabetes Endocrinol 2014;2:369-84.

37. Perkovic V, de Zeeuw D, Mahaffey KW, Fulcher G, Erondu N, Shaw W, et al. Canagliflozin and renal outcomes in type 2 diabetes: results from the CANVAS program randomised clinical trials. Lancet Diabetes Endocrinol 2018;6:691-704.

38. DeFronzo RA, Lewin A, Patel S, Liu D, Kaste R, Woerle $\mathrm{HJ}$, et al. Combination of empagliflozin and linagliptin as second-line therapy in subjects with type 2 diabetes inadequately controlled on metformin. Diabetes Care 2015;38: 384-93.

39. Lewin A, DeFronzo RA, Patel S, Liu D, Kaste R, Woerle 
$\mathrm{HJ}$, et al. Initial combination of empagliflozin and linagliptin in subjects with type 2 diabetes. Diabetes Care 2015;38:394402.

40. Wang Z, Sun J, Han R, Fan D, Dong X, Luan Z, et al. Efficacy and safety of sodium-glucose cotransporter-2 inhibitors versus dipeptidyl peptidase-4 inhibitors as monotherapy or add-on to metformin in patients with type 2 diabetes mellitus: a systematic review and meta-analysis. Diabetes Obes Metab 2018;20:113-20.

41. Zelniker TA, Braunwald E. Mechanisms of cardiorenal effects of sodium- glucose cotransporter 2 inhibitors: JACC state-of-the-art review. J Am Coll Cardiol 2020;75:422-34.

42. Heerspink HJ, Perkins BA, Fitchett DH, Husain M, Cherney DZ. Sodium glucose cotransporter 2 inhibitors in the treatment of diabetes mellitus: cardiovascular and kidney effects, potential mechanisms, and clinical applications. Circulation 2016;134:752-72.

43. Makdissi A, Ghanim H, Vora M, Green K, Abuaysheh S, Chaudhuri A, et al. Sitagliptin exerts an antinflammatory action. J Clin Endocrinol Metab 2012;97:3333-41.

44. Kanasaki K, Shi S, Kanasaki M, He J, Nagai T, Nakamura Y, et al. Linagliptin-mediated DPP-4 inhibition ameliorates kidney fibrosis in streptozotocin-induced diabetic mice by inhibiting endothelial-to-mesenchymal transition in a therapeutic regimen. Diabetes 2014;63:2120-31.

45. Shah Z, Kampfrath T, Deiuliis JA, Zhong J, Pineda C, Ying $Z$, et al. Long-term dipeptidyl-peptidase 4 inhibition reduces atherosclerosis and inflammation via effects on monocyte recruitment and chemotaxis. Circulation 2011;124:2338-49.

46. Kubota Y, Miyamoto M, Takagi G, Ikeda T, KirinokiIchikawa S, Tanaka K, et al. The dipeptidyl peptidase-4 inhibitor sitagliptin improves vascular endothelial function in type 2 diabetes. J Korean Med Sci 2012;27:1364-70.

47. Koska J, Sands M, Burciu C, D’Souza KM, Raravikar K, Liu J, et al. Exenatide protects against glucose- and lipid-induced endothelial dysfunction: evidence for direct vasodilation effect of GLP-1 receptor agonists in humans. Diabetes 2015;64:2624-35.

48. Rieg T, Gerasimova M, Murray F, Masuda T, Tang T, Rose $M$, et al. Natriuretic effect by exendin-4, but not the DPP-4 inhibitor alogliptin, is mediated via the GLP-1 receptor and preserved in obese type 2 diabetic mice. Am J Physiol Renal Physiol 2012;303:F963-71.

49. Muskiet MHA, Tonneijck L, Smits MM, van Baar MJB, Kramer MHH, Hoorn EJ, et al. GLP-1 and the kidney: from physiology to pharmacology and outcomes in diabetes. Nat
Rev Nephrol 2017;13:605-28

50. Hubers SA, Wilson JR, Yu C, Nian H, Grouzmann E, Eugster $\mathrm{P}$, et al. DPP (dipeptidyl peptidase)-4 inhibition potentiates the vasoconstrictor response to NPY (neuropeptide Y) in humans during renin-angiotensin-aldosterone system inhibition. Hypertension 2018;72:712-9.

51. Mayer GJ, Wanner C, Weir MR, Inzucchi SE, Koitka-Weber A, Hantel S, et al. Analysis from the EMPA-REG OUTCOME® trial indicates empagliflozin may assist in preventing the progression of chronic kidney disease in patients with type 2 diabetes irrespective of medications that alter intrarenal hemodynamics. Kidney Int 2019;96:489-504.

52. Wiviott SD, Raz I, Bonaca MP, Mosenzon O, Kato ET, Cahn A, et al. Dapagliflozin and cardiovascular outcomes in type 2 diabetes. N Engl J Med 2019;380:347-57.

53. Zelniker TA, Wiviott SD, Raz I, Im K, Goodrich EL, Bonaca MP, et al. SGLT2 inhibitors for primary and secondary prevention of cardiovascular and renal outcomes in type 2 diabetes: a systematic review and meta-analysis of cardiovascular outcome trials. Lancet 2019;393:31-9.

54. Nauck M. Incretin therapies: highlighting common features and differences in the modes of action of glucagon-like peptide-1 receptor agonists and dipeptidyl peptidase- 4 inhibitors. Diabetes Obes Metab 2016;18:203-16.

55. Nauck MA, Meier JJ. Management of endocrine disease: are all GLP-1 agonists equal in the treatment of type 2 diabetes? Eur J Endocrinol 2019;181:R211-34.

56. Vidakovic M, Grdovic N, Dinic S, Mihailovic M, Uskokovic A, Arambasic Jovanovic J. The importance of the CXCL12/ CXCR4 axis in therapeutic approaches to diabetes mellitus attenuation. Front Immunol 2015;6:403.

57. Takashima S, Fujita H, Fujishima H, Shimizu T, Sato T, Morii T, et al. Stromal cell-derived factor-1 is upregulated by dipeptidyl peptidase-4 inhibition and has protective roles in progressive diabetic nephropathy. Kidney Int 2016;90:783-96.

58. Jackson EK, Zhang Y, Gillespie DD, Zhu X, Cheng D, Jackson TC. SDF-1 $\alpha$ (stromal cell-derived factor $1 \alpha$ ) induces cardiac fibroblasts, renal microvascular smooth muscle cells, and glomerular mesangial cells to proliferate, cause hypertrophy, and produce collagen. J Am Heart Assoc 2017; 6:e007253.

59. Ussher JR, Drucker DJ. Cardiovascular biology of the incretin system. Endocr Rev 2012;33:187-215.

60. Mulvihill EE, Drucker DJ. Pharmacology, physiology, and mechanisms of action of dipeptidyl peptidase-4 inhibitors. Endocr Rev 2014;35:992-1019. 


\section{Appendix S1. Study protocol}

1. Title: Comparative renal effects of dipeptidyl peptidase- 4 inhibitors and sodium-glucose cotransporter 2 inhibitors on individual outcomes in patients with type 2 diabetes: a systematic review and network meta-analysis of randomized controlled trials

2. Objectives: This study was performed to compare the renal effects of dipeptidyl peptidase-4 (DPP-4) inhibitors and sodium-glucose cotransporter 2 (SGLT2) inhibitors on individual outcomes in patients with type 2 diabetes.

3. Protocol and registration: Methods of database search, study selection, data extraction, assessment of study quality and risk of bias, data synthesis, and statistical analysis were prespecified in the protocol at the beginning of the study.

4. Reporting: This systematic review and network meta-analysis was reported in accordance with the Preferred Reporting Items for Systematic Review and Meta-analyses (PRISMA) extension statement for reporting systematic reviews incorporating network meta-analyses.

\section{Eligible criteria}

\subsection{Study characteristics}
A. Population: patients with type 2 diabetes
B. Intervention: DPP-4 inhibitors or SGLT2 inhibitors
C. Control: placebo and/or other antidiabetic drugs
D. Outcomes of interests

(a) Development of microalbuminuria: defined as urine albumin-to-creatinine ratio (UACR) $>30 \mathrm{mg} / \mathrm{g}$

(b) Development of macroalbuminuria: defined as UACR $>300 \mathrm{mg} / \mathrm{g}$

(c) Worsening nephropathy: defined as the development of microalbuminuria or macroalbuminuria from normoalbuminuria, or progression from microalbuminuria to microalbuminuria

(d) Development of end-stage kidney disease (ESKD): defined as kidney failure, initiation of renal replacement therapy, or kidney transplantation

E. Study design: randomized controlled trial (RCT)

F. Study duration: 12 weeks or longer

\subsection{Report characteristics}

A. Years considered: initially published from inception to September 2017, but later extended until June 2019

B. Language: no restriction of language

C. Publication status: full-text articles with no limitation of publication status

\subsection{Inclusion and exclusion criteria}

A. The study population comprised men and women with type 2 diabetes.

B. We included RCTs comparing the efficacy or safety of DPP-4 inhibitors or SGLT2 inhibitors with placebo and/or other antidiabetic drugs.

C. We included RCTs with a duration of 12 weeks or longer.

D. We included RCTs reporting at least one kidney outcome, including UACR, estimated glomerular filtration rate (eGFR), microalbuminuria, macroalbuminuria, doubling of serum creatinine, kidney failure, ESKD, renal replacement therapy, dialysis, or kidney transplantation.

E. In duplicates or extensions, we only included a study with a longer duration or more information regarding kidney outcomes.

F. Pooled analysis or secondary analysis was included only when it provided more information regarding kidney outcomes than original publications. 
G. There was no restriction of language.

H. We included full-text articles with no limitation of publication status.

6. Information sources: We searched the electronic databases of MEDLINE, Embase, and the Cochrane Central Register of Controlled Trials.

7. Search strategy: RCTs of DPP-4 inhibitors or SGLT2 inhibitors in patients with type 2 diabetes were searched using the following search terms.

7.1. DPP-4 inhibitors

A. MEDLINE: (DPP-4 inhibitor OR DPP4 inhibitor OR alogliptin OR anagliptin OR evogliptin OR gemigliptin OR linagliptin OR omarigliptin OR saxagliptin OR sitagliptin OR teneligliptin OR trelagliptin OR vildagliptin) AND (random* OR RCT OR RCTs)

B. Embase: (DPP-4 inhibitor OR DPP4 inhibitor OR alogliptin OR anagliptin OR evogliptin OR gemigliptin OR linagliptin OR omarigliptin OR saxagliptin OR sitagliptin OR teneligliptin OR trelagliptin OR vildagliptin) AND (random* OR RCT*)

C. The Cochrane Central Register of Controlled Trials: DPP-4 inhibitor OR DPP4 inhibitor OR alogliptin OR anagliptin OR evogliptin OR gemigliptin OR linagliptin OR omarigliptin OR saxagliptin OR sitagliptin OR teneligliptin OR trelagliptin OR vildagliptin

\subsection{SGLT2 inhibitors}

D. MEDLINE: (SGLT2 inhibitor OR SGLT-2 inhibitor OR canagliflozin OR dapagliflozin OR empagliflozin OR ertugliflozin OR ipragliflozin OR luseogliflozin OR remogliflozin OR sergliflozin OR tofogliflozin) AND (random* OR RCT OR RCTs)

E. Embase: (SGLT2 inhibitor OR SGLT-2 inhibitor OR canagliflozin OR dapagliflozin OR empagliflozin OR ertugliflozin OR ipragliflozin OR luseogliflozin OR remogliflozin OR sergliflozin OR tofogliflozin) AND (random* OR RCT*)

F. The Cochrane Central Register of Controlled Trials: SGLT2 inhibitor OR SGLT-2 inhibitor OR canagliflozin OR dapagliflozin OR empagliflozin OR ertugliflozin OR ipragliflozin OR luseogliflozin OR remogliflozin OR sergliflozin OR tofogliflozin

8. Study selection: All identified records were independently screened and evaluated for eligibility by two reviewers. The titles, abstracts, and full texts of the studies were thoroughly reviewed. Any disagreements were resolved by consensus among investigators of the study.

9. Data extraction: Standardized data extraction was performed independently by two reviewers as follows. Any discrepancies were resolved by consensus among investigators of the study.

9.1. First author

9.2. Publication year

9.3. Intervention

9.4. Comparator

9.5. Number of participants in the analysis

9.6. Age of participants

9.7. Study duration

9.8. Duration of diabetes mellitus

9.9. Background antidiabetic drugs

9.10. Baseline eGFR

9.11. Number of participants reporting microalbuminuria

9.12. Number of participants reporting macroalbuminuria 
9.13. Number of participants reporting worsening nephropathy

9.14. Number of participants reporting ESKD

10. Assessment of study quality and risk bias: We assessed quality and risk of bias of the studies using the Cochrane Risk of Bias Tool. Two reviewers independently evaluated each study according to the following aspects of trials.

10.1. Random sequence generation

10.2. Allocation concealment

10.3. Blinding of participants and personnel

10.4. Blinding of outcome assessment

10.5. Incomplete outcome data

10.6. Selective reporting

10.7. Other sources of bias

\section{Data synthesis}

11.1. Network geometry: Geometry of the network of included studies is presented graphically using nodes and lines.

11.2. Network meta-analysis: We conducted pairwise meta-analyses using a fixed effect model to estimate the effect size of each treatment. We performed an arm-based network meta-analysis for evaluating individual kidney outcomes using Bayesian methods and reported results as median odds ratios and their $95 \%$ credible intervals.

A. Subgroup analysis: We conducted a prespecified subgroup analysis to assess the effects of treatments on ESKD for studies with a duration of 52 weeks or longer.

11.3. Absolute risks of the treatments: We calculated the posterior densities of absolute risks of treatments for each kidney outcome.

11.4. Rank probabilities: We evaluated relative rank probabilities to rank the best treatments for each kidney outcome.

11.5. Statistical heterogeneity: We used the $I^{2}$ statistic, $\tau^{2}$ statistic, and Cochran's $Q$ test for testing statistical heterogeneity.

11.6. Checking inconsistency: We checked inconsistency of direct and indirect estimates using a back-calculation method with a fixed effect model. 